IEEE Transactions on Nuclear Science, Vol. NS-28, No. 3, June 1981

\title{
A PULSE TO PULSE KLYSTRON DIAGNOSIS SYSTEM*
}

J. Nowak, V. Davidson, L. Genova, R. Johnson and D. Reagan

Stanford Linear Accelerator Center

Stanford University, Stanford, California 94305

\section{Introduction}

This report will describe a system used to study the behavior of SLAC high powered klystrons operating with a twice normal pulse width of $5 \mu \mathrm{s} .5$ At present, up to eight of the klystrons installed along the accelerator can be operated with long pulses and monitored by this system. The report will also discuss some of the recent findings and investigations.

\section{Fault Detection}

The modulator-klystron protection $\operatorname{logic}^{1}$ was designed to provide performance monitoring, protection, and fault detection during normal klystron operation. Effectively evaluating long-pulse operation requires signals not provided by this standard equipment. The new signals must be capable of responding within microseconds thereby enabling the detection of single pulse faults (those which affect only one pulse of electrons). Some of the fault and status signals that were found to provide the most information on klystron and modulator operation are presented in Table $I$.

An interface chassis was designed that provides isolation, level translation, buffering, and latching of the existing status and fault data. It also provides for monitoring six new signals. This interface supplies as output six buffered analog signals, eighteen digital status signals, and a trigger signal that occurs approximately .5 microsecond prior to the klystron pulse. Typical circuits are shown in Fig. 1.

\section{Data Acquistion Requirements}

The data acquisition system must detect specificd faults and save all pertinent data within $5.6 \mathrm{mfllise-}$

\section{Table I}

Name

Average klystron beam current $\mathrm{RF}$ drive attenuator position Long recycle

Waveguide vacuum

High voltage supply current

End of line clipper averaged current

End of line clipper pulse current

Thyratron trigger current

Klystron beam voltage pulse

Klystron beam current pulse

Klystron forward RF power

Klystron reflected $R F$ power

Forward RF power from SLED cavity

Reflected RF power from load

RF drive level

Fault Condition

Low

Inserted

Active

High

High

High

High

High

High

High

Low or narrow pulse

High

Low or narrow pulse High

Low or narrow pulse

conds (interpulse period at 180 pps). It must also record the duration of the fault state. Finally it must be able to digitize $5 \mu$ sulses with reasonable resolution.

\section{Hardware Specification}

CAMAC" was chosen to interface the signals to the computer. This choice was made because all the funct1ons necessary to satisfy the data acquisition requirements are readily available from "off-the-shelf" CAMAC equipment. The fault and status signals are connected to standard digital input modules. Analog signals are digitized with six high speed, analog-to-
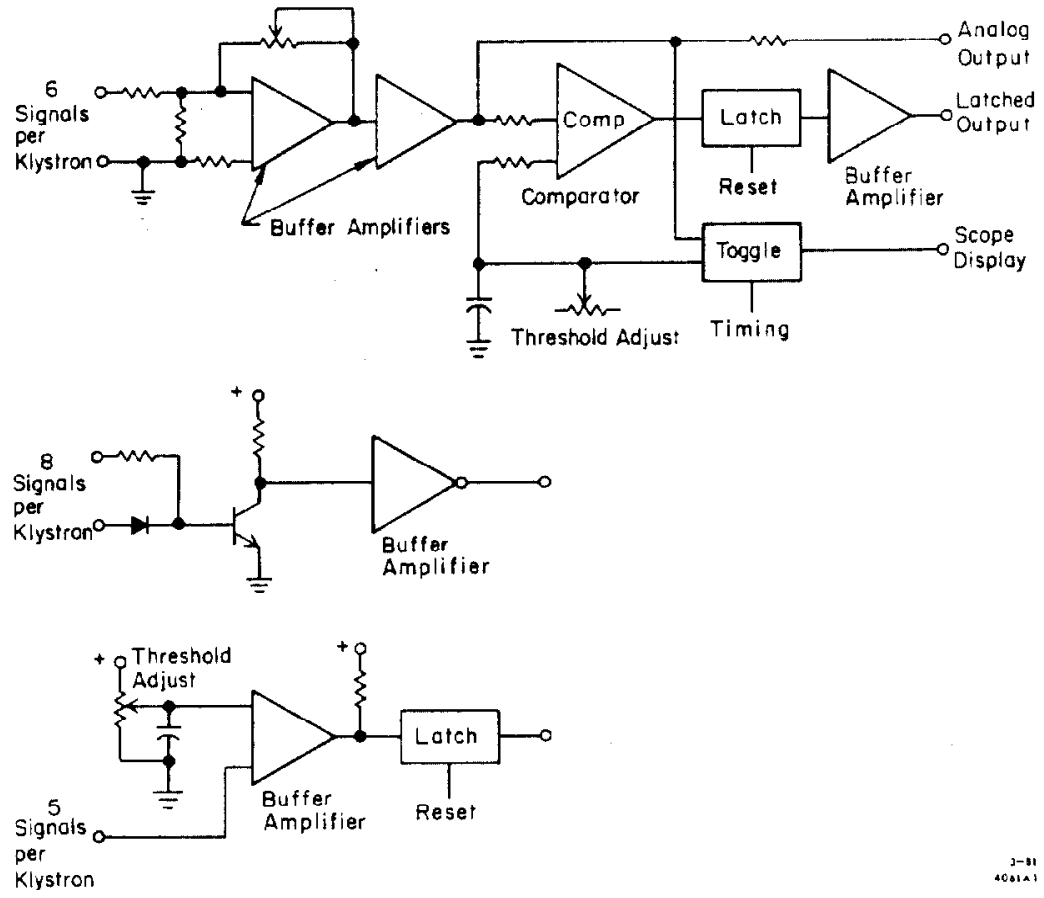

Fig. 1. Typical circuits for each of eight data acquisition packages.

* Work supported by the Department of Energy, contract DE-AC03-76SF00515.

5 Also involves lowering the maximum pulse repetition rate from 360 to 180 pulses per second.

I Computer Automated Measurement and Control. 
digital converters providing six-bit conversions at .2 us intervals. These results are stored in a 256 word memory in each digitizer. Overall control of the data acquisition process is provided by a LSI $11^{\#} \mathrm{mi-}$ crocomputer. The peripherals include four serial communication ports, a floppy disc, and a CAMAC controller interface. Long-term operation of the floppy disc in the sector instrumentation alcove would be unreliable due to heat and dust. Therefore, the floppy disc is used only to load the program memory or save the current operating conditions. Functions of data storage, analysis, and display are handled by a computer system located in the SLAC Main Control Center (MCC). The MCC computer used is a PDP $11-34^{\dagger}$. It provides for complete control of the analysis programs through the use of a touch panel. Figure 2 is a block diagram

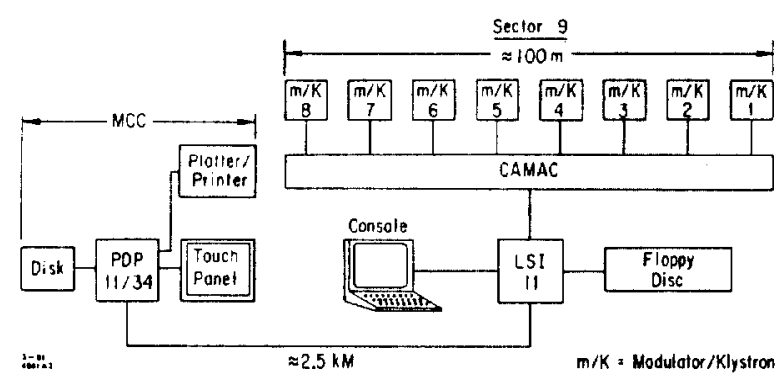

Fig. 2. Arrangement of equipment.

outlining hardware location and interconnection.

\section{Software}

The software was designed to allow the data acquisition process to be redefined with a minimum of effort. The discussion that follows will outline the data structure and present briefly how it is used. It also describes the two major microcomputer routines.

\section{Data Structure}

Eight arrays provide the data structure for the microcomputer programs. The information stored in these arrays provides ordered lists of data necessary for acquiring, identifying, and storing data. The flexibility of system configuration is provided primarily by a list of pointers; a linked list that permits an arbitrary arrangement of sequential operations to be specified easily.

\section{Monitor Program}

The monitor allows local control, modification, and testing of the data acquisition system. Functions include listing and editing of the data structure, starting and stopping of the data acquisition process, and testing of individual CAMAC modules.

\section{Data Acquisition Program}

The digitizing process is activated by a klystron trigger signal. Approximately 51.2 us later, highspeed digitizer module memories fill, and the computer is interrupted. The interrupt routine reads the status information from the eight digital input modules and masks off signals that are to be ignored for the test. If, however, an "unmasked" fault condition exists for a particular klystron, the linked list in the data structure is used to determine the data to be recorded for that event. If a fault condition existed for a klystron during the preceding pulse, further data is not recorded for that klystron unt 11 the signal returns to normal condition, thereby making it possible to determine the time it takes for a fault to clear.

The packet of information transmitted to the computer in MCC is composed of the status information, mask information, any data acquired through the linked list, and the time, to the nearest. 1 sec., that the data was recorded. This data is stored on disk in MCC and can be reviewed via a touch panel. The touch panel provides waveform displays and summary report functions.

\section{Results}

The testing to date has provided some unexpected results. Breakdown of the output RF pulse occurs at $a$ much higher rate than previously suspected. This condition seems to exist even during short pulse operation and manifests itself as beam instability. The origin of the beam instability was hidden by an inability to detect single pulse RF faults. We are currently conducting a study in which klystron voltage, pulse length, and pulse repetition rate are varied. This procedure should indicate an optimum set of operating conditions for the present system. Examples of faulted and normal RF pulse waveforms are shown in Fig. 3 .

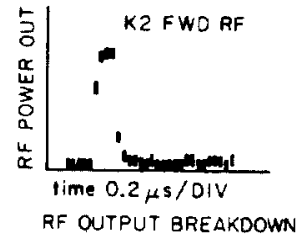
3-a।

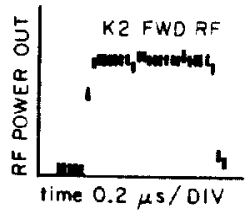

NORMAL RF PULSE
Fig. 3. Two pulses recorded by a high-speed digitizer.

\section{Conclusions}

This system is a useful tool for the analysis of klystron behavior and because of this, certain aspects may be incorporated into future klystron monitoring and protection improvements. Usefulness has been directly related to configuration flexibility. Reassignment of high-speed digitizers and mask revisions can be made in a matter of minutes. Overall, the system has been very useful in monitoring operational trends and in guiding efforts to improve kIystron operation.

\section{Reference}

1. R. B. Neal, ed., The Stanford Two-Mile Accelerator (Benjamin, New York, 1968) pp. 498-500. 